\title{
Trapped-Ion Cell with Improved DC Potential Harmonicity for FT-ICR MS
}

\author{
Aleksey V. Tolmachev, ${ }^{a}$ Errol W. Robinson, ${ }^{a}$ Si Wu, Hyuk Kang, ${ }^{\text {b }}$ \\ Natacha M. Lourette, ${ }^{a}$ Ljiljana Paša-Tolić, ${ }^{a}$ and Richard D. Smith ${ }^{a}$ \\ a Biological Sciences Division, Pacific Northwest National Laboratory, Richland, Washington, USA \\ b Department of Chemistry, Ajou University, Suwon 443-749, Korea
}

\begin{abstract}
The trapped-ion cell is a key component critical for optimal performance in Fourier transform ion cyclotron resonance (FT-ICR) mass spectrometry (MS). To extend the performance of FT-ICR MS, we have developed a new cell design that is capable of generating a DC trapping potential which closely approaches that of an ideal Penning trap, i.e., a 3D axial quadrupolar potential distribution. The new cell design was built upon an open cylindrical geometry, supplemented with two pairs of cylindrical compensation segments. Electric potential calculations for trial cell geometries were aimed at minimizing spatial variations of the radial electric field divided by radius. The resulting cell proportions and compensation voltages delivered practically constant effective ion cyclotron frequency that was independent of ion radial and axial positions. Our customized 12 tesla FT-ICR instrument was upgraded with the new cell, and the performance was characterized for a range of ion excitation power and ion populations. Operating the compensated cell at increased postexcitation radii, $\sim 0.7$ of the cell inner radius, resulted in improved mass measurement accuracy together with increased signal intensity. Under these same operating conditions the noncompensated open cell configuration exhibited peak splitting and reduced signal life time. Mass accuracy tests using 11 calibrants covering a wide $\mathrm{m} / \mathrm{z}$ range reproducibly produced under $0.05 \mathrm{ppm}$ RMS precision of the internal calibration for reduced ion populations and the optimal excitation radius. Conditions of increased ion population resulted in a twofold improvement in mass accuracy compared with the noncompensated cell, due to the larger achievable excitation radii and correspondingly lower space charge related perturbations of the calibration law. (J Am Soc Mass Spectrom 2008, 19, 586-597) (c) 2008 American Society for Mass Spectrometry
\end{abstract}

$\mathrm{F}$ ourier transform ion cyclotron resonance (FTICR) mass spectrometry (MS) is an advanced mass analyzer technology, which has been at the leading edge in terms of mass resolving power and mass measurement accuracy since the early years of its inception [1]. A number of reviews describe the general features of FT-ICR MS and the milestones of its historical evolution, as well as the current rapid expansion of its use in biomolecule analysis and, in particular, proteomics [2-8]. Although the main components of FTICR MS are well established, the growing complexity of modern applications creates a driving force for further optimizations. Fine-tuning acquisition time, excitation power (i.e., excitation radius), trapping voltage, and the type of excitation waveform can significantly improve both mass measurement accuracy and quantitation accuracy of FT-ICR measurements [9, 10].

A key component of the FT-ICR mass analyzer is the trapped-ion cell (ICR cell, ICR trap, Penning trap) where measurement of the mass-to-charge ratio $(\mathrm{m} / \mathrm{z})$ of an ion takes place. Within the trapped-ion cell, each

Address reprint requests to Dr. Ljiljana Paša-Tolić, Biological Sciences Division, Pacific Northwest National Laboratory, P.O. Box 999, Richland, WA 99352. E-mail: ljiljana.pasatolic@pnl.gov measurement cycle requires ion injection, excitation, and acquisition of the induced ion signal (i.e., ion transient detection). Historically, closed orthorhombic cells, predominant in early FT-ICR mass spectrometers, were replaced by cylindrical cells with either capacitive coupling open configuration or so-called infinity cell layout, i.e., a closed cell with trapping plates that have excitation circuitry that emulates axially-infinite distribution of the excitation field [11-14].

It is well recognized that deviations from the ideal quadrupolar DC potential, which is characteristic for such cell geometries, impose deleterious effects on FT-ICR measurements and various approaches to the cell anharmonicity problem have been explored [14-27]. A thorough study [14] considered various FT-ICR cell designs optimizing the three cell functions, ion trapping, excitation and detection, and suggested as a possible solution the matrix-shimmed cell. Such a cell was build in a form of a cubic cell with each of the six sides split into a matrix of $5 \times 5$ electrodes, 150 electrodes total, and tested experimentally [22]. This previous study reported elimination of harmonic peaks and suggested cell performance could be significantly improved with an idealized 3D trapping potential. The cyclotron frequency variation with excitation radius 
was suppressed and no peak splitting was evident at increased excitation radii where the cubic cell produced a significant peak splitting [22].

An alternative solution was proposed in a form of open cylindrical cell having 32 poles for ion excitation and detection and 15 cylindrical ring electrodes to generate trapping potential wells [24]. It was demonstrated that ions can be successfully trapped and detected with the 32-pole cell, and performance was characterized for different trapping well shapes. Although both cell designs have been successfully tested experimentally, neither is currently in use in the field of FT-ICR bio-molecular applications, and additional studies are needed to determine if these radically changed cell layouts can be suited for practical implementation.

We have revisited the problem of the DC potential harmonicity of the ICR cell in the context of MS proteomics studies, where common attributes of FT-ICR measurements, such as high resolving power, mass measurement accuracy, precision, dynamic range, and sensitivity, all should be realized reliably and reproducibly under conditions of high-throughput. We recently reported a study of mass accuracy trends, showing the necessity to simultaneously account for several factors that influence mass measurements due to the highly variable conditions in high-throughput LC FT-ICR MS, which led to the implementation of multidimensional recalibration for such datasets $[28,29]$. Results of this earlier work indicated that along with total ion current (TIC) variations, the variability of the ion abundance across the mass spectrum was detrimental to mass measurement accuracy.

Current high-throughput methods often employ external accumulation of ions followed by gas-free injection and trapping in the ICR cell. Such approaches eliminate the cell pump-down delays, which improve duty cycle. However, as a side effect, accurate mass measurements in FT-ICR become more sensitive to the "local" ion abundance variability. The variations of the axial kinetic energy of ions ejected from the external RF multipole [30-32] results in a variable and mass-dependent axial distribution of ions during FT-ICR excitation and detection. This variation in axial kinetic energy and axial density distribution of ions results in varied cyclotron frequency shifts due to the nonuniform radial electric fields present in all cell geometries [33]. The mass measurement accuracy is also influenced by other conditions such as excitation power (i.e., postexcitation radius) and trapping voltage [9, $10,32,34-36]$. A cylindrical cell with grids as excitation electrodes that extend past the region in which the ions are trapped was recently reported by Wieghaus et al. [36]. Their study demonstrated increased ion excitation radii were achievable with the new cell design, resulting in improved ion signal intensity and mass measurement accuracy. The grid cell was chosen as the new standard cell for ThermoFinnigan LTQ-FT Ultra hybrid FTICR instrument [36].

Generally, finding a stable and sample-independent optimum for automated high-throughput FT-ICR MS operation represents a significant challenge. Minimiz- ing the spatial variability of the radial electric field should significantly improve the stability of optimal operating conditions, which will improve mass measurement accuracy achievable in LC FT-ICR MS.

The open cylindrical cell design is currently widely used in FT-ICR MS and has a long history of successful applications. Here, we report correcting the open cell trapping potential to make it closer to the three-dimensional (3D) axial quadrupolar electrostatic potential distribution. Using numerical potential calculations, we searched for a configuration that does not excessively complicate the cell design while providing significant, at least an order of magnitude, improvement of the cell harmonicity. We adopted an open cylindrical configuration with added compensation electrodes, based on the pioneering work by Gabrielse et al. [17]. Their study demonstrated that by adding the compensation electrodes to the open cylindrical cell, anharmonicities of the trapping potential could be tuned out, thus enabling the high precision measurements [17]. A similar approach was later implemented in the screened open cell design [20], which used a pair of cylindrical compensation segments to minimize magnetron frequency at the cell center midplane (i.e., for ions that have negligible axial kinetic energy). The cell operation regime of interest for us involved ions having distributed axial positions that correspond to a range of kinetic energies typical for gas-free ion injection $(\sim 1 \mathrm{eV})$. Proportions of the cell segments were determined based on minimization of the spatial variations of the radial electric field divided by radius, the quantity that determines the ion magnetron frequency [3]. An acceptable configuration was found in a form of the cell with two pairs of compensation segments having certain geometrical proportions and specific compensation voltages. We report a description of the new cell configuration and properties of its trapping potential and then provide experimental characterization results.

\section{Methods}

\section{Cell Design}

An extensive and detailed treatment of the electric field configurations and corresponding ICR cell designs has been previously reported [14]. Below we provide a concise consideration focused on the cell harmonicity optimization employed in the present work.

The ideal 3D quadrupolar DC potential can be expressed as follows [14-17]:

$$
V(r, z)=V_{0}\left(z^{2} / a^{2}-r^{2} / 2 a^{2}\right)+C
$$

Here, the cylindrical coordinate system $(r, z)$ is assumed with radius $r=\left(x^{2}+y^{2}\right)^{0.5}$, axial coordinate $z$, measure of the trap size $a$, and constant $C$. Such an ideal quadrupolar potential can be realized in a Penning trap. The electrodes of such a trap are shaped according to equipotential surfaces of the ideal potential distribution 

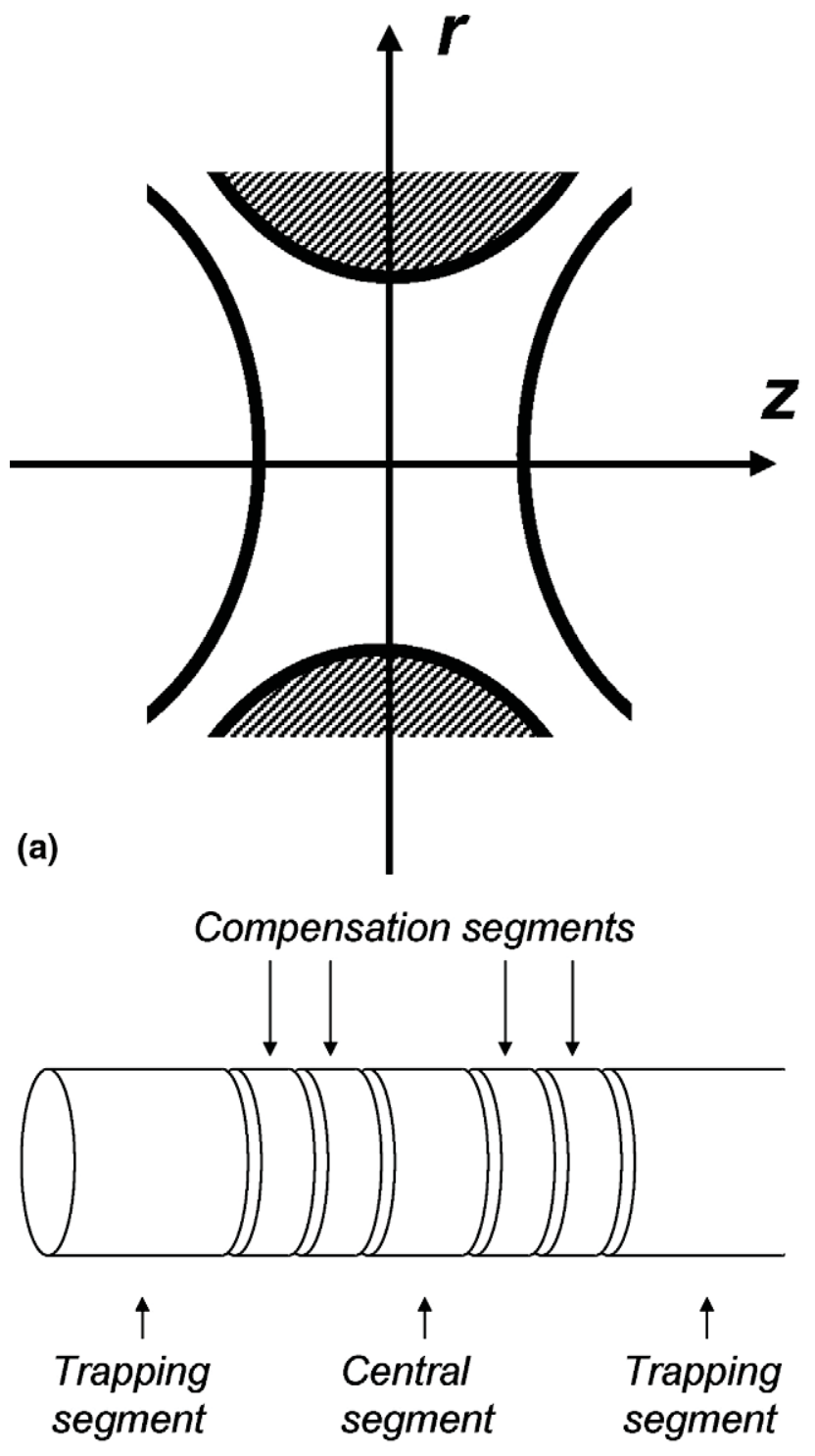

(b)

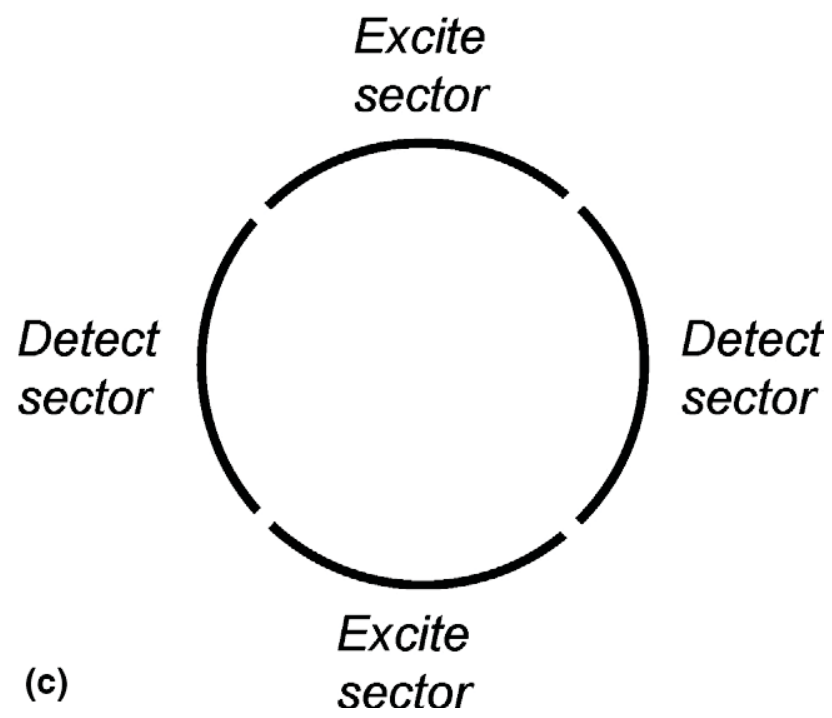

$V(r, z)$, Figure 1a. The equipotential surfaces are defined by the following equations:

$$
\begin{array}{ll}
V(r, z)=V_{\text {trap }} & \text { for end caps } \\
V(r, z)=V_{\text {ring }} & \text { for ring electrodes }
\end{array}
$$

The cell geometry, i.e., radius $\left(r_{0}\right)$ and distance from cell center to the end of the cell $\left(z_{0}\right)$, determine how $V_{0}, C$, and $a$ values in eq 1 are related to $V_{\text {trap }}$ and $V_{\text {ring }}$ of eq 2 .

The resulting ion motion in the ideal potential (eq 1), assuming a homogeneous magnetic field $\left(B_{0}\right)$ directed along the $z$-axis and negligible space charge effects, is a rotation with reduced cyclotron frequency $\omega_{+}$and with the guiding center drifting at the magnetron frequency $\omega_{-}$. The frequency shift $(d \omega)$ due to the trapping radial electric field $\left(E_{r}\right)$ is equal to the magnetron frequency $\omega_{-}$. It is convenient to apply first-order approximation to obtain simple relationships for $\omega_{-}, d \omega$, and $\omega_{+}[37,38]$ :

$$
\begin{aligned}
& \omega_{-}=d \omega=E_{r} / r B_{0} \\
& \omega_{+}=\omega_{c}-d \omega
\end{aligned}
$$

Practically, the frequency shift $d \omega$ is much smaller than the observed cyclotron frequency $\omega_{+}$and the first-order relationships eqs 3 and 4 are quite accurate. The unperturbed cyclotron frequency can be expressed via the $\mathrm{m} / \mathrm{z}$ value commonly used in mass spectrometry:

$$
\omega_{c}=\frac{e B_{0}}{m_{u} m / z}
$$

Here SI units are assumed for $B_{0}$, elementary charge $e$ equals $1.60217733 \times 10^{-19} \mathrm{C}$ and unit mass $m_{u}$ is $1.66053873 \times 10^{-27} \mathrm{~kg}$.

The ratio of the radial field $E_{r}$ to radius $r$ can be obtained by taking a derivative of potential (eq 1) over radius:

$$
E_{r} / r=V_{0} / a^{2}
$$

and using eq 3 to obtain:

$$
d \omega=\frac{V_{0}}{a^{2} B_{0}}
$$

It follows that the frequency shift due to the radial electric field $E_{r}$ is constant, independent of the radial or

Figure 1. (a) An ideal Penning trap configuration corresponding to equipotential contours of the ideal 3D quadrupolar DC potential, eq 1 . The compensated cell configuration with $(\mathbf{b})$ the central cylindrical segment and two trapping segments supplemented by four compensation electrodes and (c) each segment split into four sectors, two for excitation and two for detection. 
axial position, which is a well known property of the ideal quadrupolar potential. This potential distribution is ideal for FT-ICR mass measurements because the effective ion cyclotron frequencies are stable and independent of radial and axial coordinates. The two classical FT-ICR calibration functions are very accurate in the case of the ideal harmonic potential [38]:

$$
\begin{aligned}
& m / z=A_{L} / f+B_{L} / f^{2} \\
& m / z=A_{F} /\left(f+B_{F}\right)
\end{aligned}
$$

Here $f=\omega_{+} / 2 \pi$ is the experimentally observed effective ion cyclotron frequency. Ledford's calibration function, eq $8 \mathrm{a}$, has calibration coefficients $A_{L}, B_{L}$, and Franc's calibration function, eq $8 \mathrm{~b}$, has calibration coefficients $A_{F}, B_{F}$ [37-39].

While the Penning trap geometry was explored using ion cyclotron resonance in physics experiments $[40,41]$, the ideal geometry was not utilized in FT-ICR MS, due to the inefficiency of the Penning trap in terms of using the magnet bore volume compared with other geometries, including cylindrical cells (i.e., small ion capacity), unfavorable ion excitation configuration, etc. [14]. While the cylindrical cell efficiently utilizes the magnet bore volume, there is significant variability in the radial electric field per radius $\left(E_{r} / r\right)$ in both radial and axial dimensions. These deviations from the ideal cell potential can be minimized with compensation ("shim" or "guard") electrodes and optimization of cell proportions [14-27]. A possible approach for finding dimensions and potentials of the compensation electrodes is to create a system of equations that define ideal conditions in a number of spatial locations, assumed to be important for the cell operation. Alternative approaches can be based on power series expansions of the potential or ion motion characteristics. Some formal approaches we explored had a tendency to produce impractical dimensions or potentials of the compensation electrodes and unstable electric field behavior, e.g., at locations other than those used for optimization.

Under the present cell optimization approach, we relied on the property of the Laplace equation to smooth, in a diffusion-like fashion, small perturbations of the boundary conditions. This smoothing property results in the potential distribution of a small region around the center of a noncompensated cell to closely approach the ideal behavior. Here, we aimed to extend such a paraxial region by using a system of compensation electrodes added to the open cylindrical cell geometry.

We have considered a number of trial configurations, using potential calculations to evaluate the spatial nonuniformity of the radial electric field per radius, $E_{r} / r$, which is responsible for the cyclotron frequency shift (eq 3). A selected configuration (Figure $1 \mathrm{~b}$ and c) was found to provide an acceptable combination of improved potential harmonicity and a reasonably simple physical geometry. This configuration had a central
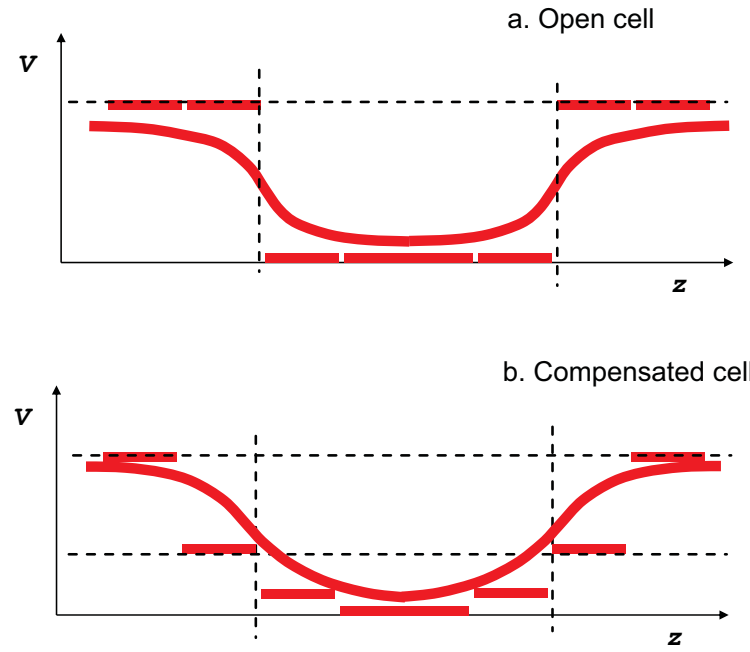

Figure 2. The potentials applied to the cell segments correspond to two configurations used for the experimental characterization: (a) the typical open cell configuration with the DC offsets of the compensation electrodes set to 0 for the inner pair and to the trapping potential for the outer pair, and (b) the compensated cell configuration with the compensation segment potentials set to optimum values to minimize deviations from the potential distribution of the ideal Penning trap.

segment and a trapping segment at either end, as in typical open cylindrical cells. A pair of short compensation segments was added between the central and each trapping segment. All segments had the same internal diameter $(D)$ of $60 \mathrm{~mm}$ and the lengths (z-axis) chosen for the 7 cylindrical segments were 1, 0.2, 0.2, $0.5,0.2,0.2,1$, in units of the cell internal diameter, $D$. Potential calculations for such cell proportions produced the following DC offsets that delivered close to harmonic potential distribution: 1, 0.3167, 0.1333, 0, 0.1333, 0.3167, 1 (in relative units). Each cylindrical segment was split into four sectors, two for detection and two for excitation, and capacitive coupling was applied to the excitation sectors of all segments. Ion transient detection was acquired at the two detection sectors of the central segment. (Detection on compensation electrodes could also be realized, but will not be reported here.) Setting the DC offsets of the compensation electrodes closest to the central segment to $0 \mathrm{~V}$ and those closest to the trapping segments to the trapping potential of the cell (Figure 2a) resulted in a potential distribution typical of the open cylindrical cell. The cell aspect ratio was 0.9 for this configuration, similar to the aspect ratio of the Bruker Infinity cell; Bruker Daltonics, Billerica, MA. From symmetry considerations, the potential distribution inside a closed cylindrical cell approaches the potential near the center of an open cell of the same aspect ratio when the trapping voltage of the closed cell is half that of the open cell. This consideration implies that the potential distribution inside the open cell should approach the potential distribution inside the Bruker Infinity cell with $2 \times$ lower trapping voltage. 

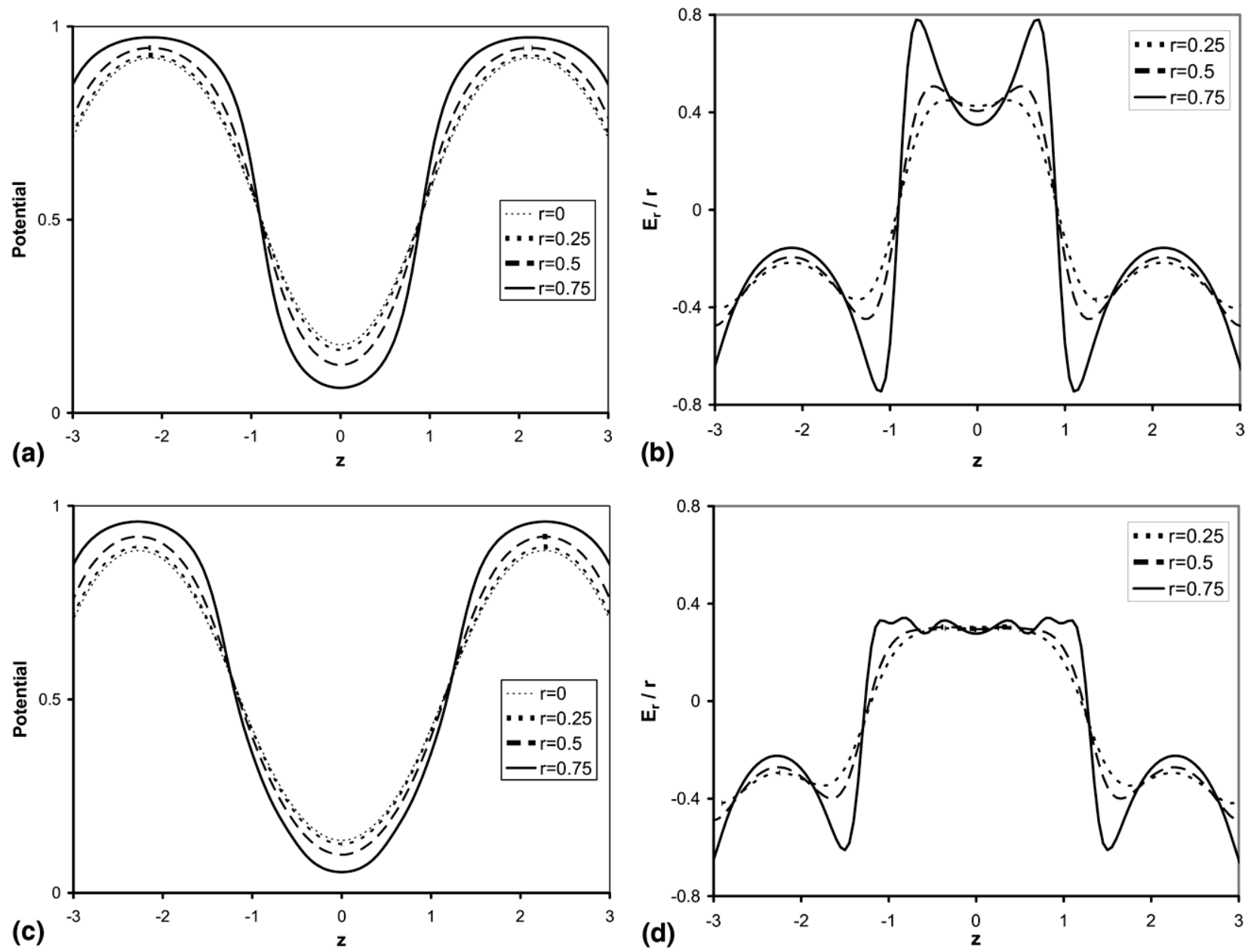

Figure 3. Potential calculation plots for the two cell voltage configurations with units of length equal to the cell radius $r_{0}$ : (a) potential distribution for the open cell configuration; (b) radial electric field divided by radius, calculated for the open cell; (c) potential distribution for the compensated cell configuration; and (d) radial electric field divided by radius, calculated for the compensated cell.

Typically, the compensation segment potentials would be set to the optimized values that minimize deviations from the ideal potential distribution (Figure $2 b)$. Here, we switched between the two cell voltage configurations to demonstrate the effectiveness of the compensation electrodes; "open cell" refers to the voltage configuration closest to that of a typical cylindrical cell (Figure 2a) while "compensated cell" refers to the voltage configuration closest to the ideal potential distribution (Figure $2 b$ ).

Figure 3 shows potential $(V)$ calculations for the two cell voltage configurations that are plotted with units of length equal to the cell radius $\left(r_{0}\right)$. The different lines represent potential calculations at different radial distances from the cell axis. The potential profiles for the open cell in Figure 3a appear similar to profiles for the compensated cell in Figure 3c. The effect of compensation was more pronounced in the profiles of the radial electric field divided by radius $\left(E_{r} / r\right)$, where the $E_{r} / r$ deviations of the open cell (Figure $3 b$ ) were significantly larger than those of the compensated cell (Figure 3d). The improved spatial uniformity of $E_{r} / r$ for the compensated cell was the target of cell configuration optimization.

The following simple calculations demonstrate how variability of $E_{r} / r$ translates into variations of the observed cyclotron frequency. Assume a cell trapping voltage of $1 \mathrm{~V}$ for two values of the post-excitation radius $\left(R_{\text {exc }}\right), 0.5$ and 0.75 of the cell radius, and an ion axial kinetic energy $\left(K_{z}\right)$ distributed in the range 0 to 0.1 $\mathrm{eV}$. In the case of the open cell, $K_{z}$ of $0.1 \mathrm{eV}$ corresponds to an amplitude of axial oscillations $(d z) \sim 0.5$, as can be seen from the depth of potential wells for an $R_{\text {exc }}$ of 0.5 and 0.75 in Figure $3 a$. The corresponding $E_{r} / r$ profiles in Figure $3 \mathrm{~b}$ show variations from $0.4(z$ at 0$)$ to 0.5 ( $z$ at 0.5 ) for $R_{e x c}$ of 0.5 , and from 0.34 ( $z$ at 0$)$ to 0.64 ( $z$ at 0.5 ) for $R_{\text {exc }}$ of 0.75 . Using eq 3 and the cell radius $\left(r_{0}\right)$ of 3 $\mathrm{cm}$, the magnetron frequency shift $(d f)$ in the axial range from a $z$ of 0 to $0.5 r_{0}$ (or 0 to $1.5 \mathrm{~cm}$ ) is $1.5 \mathrm{~Hz}$ at $R_{e x c}$ of 0.5 and $4.4 \mathrm{~Hz}$ at $R_{e x c}$ of 0.75 . These variations can be compared to the cyclotron frequency using eq 5; assuming an $\mathrm{m} / \mathrm{z}$ of 1000 and magnetic field $\left(B_{0}\right)$ of 12 tesla, the unperturbed cyclotron frequency $(f c)$ is $184 \mathrm{kHz}$ (note 
$\left.f_{c}=\omega_{c} / 2 \pi\right)$. The frequency variations for the open cell would be 8 ppm for $R_{\text {exc }}$ of 0.5 and 24 ppm for $R_{\text {exc }}$ of 0.75 . For the compensated cell potential (Figure 3c), a $K_{z}$ of $0.1 \mathrm{eV}$ corresponds to z-oscillations with $d z$ of $0.6 r_{0}$ (or $1.8 \mathrm{~cm}$ ). The corresponding radial field variations $\left(\Delta E_{r} / r\right)$ are 0.001 for $R_{\text {exc }}$ of 0.5 and 0.055 for $R_{\text {exc }}$ of 0.75 (Figure $3 \mathrm{~d}$ ) and the corresponding frequency shifts are 0.08 and 4.4 ppm, a significant improvement over the open cell configuration.

The effect of $E_{r} / r$ inhomogeneity is reduced somewhat by motional averaging [33], and in practice the frequency deviations are in the $\sim 1$ to 10 ppm range. While motional averaging reduces the anharmonicity effect, ion frequencies still depend upon the spatial or kinetic energy distribution of ions. The previous estimations demonstrate that cell anharmonicity causes cyclotron frequency shifts and that the compensated cell provides up to an order of magnitude improvement in $E_{r} / r$ variations. The extent of the improvement depends on the postexcitation radius and axial range of ion motion. Importantly, the cell potential anharmonicity effects become more pronounced with increased or variable ion axial kinetic energy before excitation and during detection, which is often the case under the high-throughput LC FT-ICR operation. To demonstrate the practical significance of improved cell potential harmonicity, we present extensive data acquired for both the compensated and the open (i.e., noncompensated) cell configurations.

\section{Instrumentation}

The compensated cell was constructed from 28 goldplated copper electrode sectors mounted to a titanium support tube with o.d. $75 \mathrm{~mm}$ and length of $278 \mathrm{~mm}$. The cell was constructed to fit inside the $100 \mathrm{~mm}$ bore of the superconducting magnet and all electrode segments had i.d. $60 \mathrm{~mm}$. The widths of the trapping, compensation, and central segments were 59, 10, 28, and $28 \mathrm{~mm}$. A PEEK isolation plate was placed between each copper sector and the titanium support tube. A $2 \mathrm{~mm}$ gap was placed between electrodes to insure electrical isolation and the total length of the cell was $198 \mathrm{~mm}$. The circuit delivering DC offsets and capacitive coupling to the cell segments was similar to one described previously [42, 43] with $150 \mathrm{k} \Omega$ resistors and $330 \mathrm{pF}$ capacitors, with independently controlled DC offsets of the additional compensation electrodes. All excitation sectors of the electrode segments were capacitively coupled to the excitation RF. This configuration insured the same excitation phase on the electrode sectors on the same side of the cell. The DC offsets of the trapping and compensation electrode segments were wired so that the potential applied to the electrodes could be independently controlled outside of the vacuum chamber, which allowed for rapid conversion between the open and compensated cell electrode voltage configurations. The cell wiring scheme was extensively tested before installation of the cell in the vacuum chamber of the modified
Bruker 12 T FT-ICR mass spectrometer [44] to insure the proper DC potentials and RF excitation waveforms were applied to each electrode. LC-MS measurements were conducted using $100 \mathrm{~min}$ separation with $150 \mu \mathrm{m}$ i.d. LC column, using the mixture of 12 standard proteins digested with trypsin and 23 peptides. This solution has been previously used for quality control (QC) analyses [29] and has a total peptide concentration of $0.004 \mathrm{mg} / \mathrm{mL}$. Each LC-MS dataset contained $\sim 3000$ mass spectra, $2 \mathrm{~s}$ per spec. The corresponding accurate mass and time (AMT) tag database consisted of 4208 mass tags.

\section{Data Acquisition}

Mass spectra with up to 11 calibrant ions were acquired on a modified Bruker 12 tesla FT-ICR mass spectrometer [44], using electrospray ionization of an aqueous solution of a peptide mixture. The solution consisted of $0.1 \%$ acetic acid, $0.025 \%$ trifluro-acetic acid, and 0.5 $\mu \mathrm{g} / \mathrm{mL}$ of each of the following peptides acquired from Sigma-Aldrich (St. Louis, MO) and used as received: renin inhibitor, bradykinin, neurotensin, angiotensin, substance P, fibrinopeptide A, and G endorphin.

Mass spectra of this solution were acquired over a range of excitation powers, external accumulation times, and cell trapping voltages in automated fashion using Xmass (Bruker) scripts. Typically, excitation attenuation ranged from 0 to $20 \mathrm{~dB}$, external accumulation times were $0.05,0.1$, and $0.2 \mathrm{~s}$ and cell trapping voltages were 1 to $4 \mathrm{~V}$. External accumulation was performed in the standard Bruker hexapole with a short pulse of gas to increase trapping efficiency. Collections of spectra were processed using in-house developed software to characterize instrument performance. Each transient was zero-filled twice, Fourier-transformed, and converted to the $m / z$ domain. The mass measurement accuracy of the internal calibration was calculated as RMS of relative mass errors (column $\mathrm{dm}_{\mathrm{r}}$ in Table 1) from internal calibration of peptide ions that had ion abundances within a relative dynamic range of 10 (typically 8 to 9 calibrants). Alternative mass accuracy tests excluded a number of calibrants from internal calibration to be used as virtual unknown masses or "external calibration standards." Such tests produced on average similar mass error values, and the mass accuracy profiles versus the excitation power showed the same trends as for the first approach, which is more robust when large ranges of ion populations and excitation powers are surveyed, and varying number of calibrants detected.

The characteristic exponential life-time of the ion transients, $t_{e}$, was evaluated by fitting experimental transients to the exponential decay profile. The fit was approximate and the result depended upon the method of transient segmentation. The initial $0.5 \mathrm{M}$ interval of the transient signal (1 $\mathrm{M}$ data points total) was split into eight $64 \mathrm{k}$ segments. The data points in each transient segment were first Fourier-transformed 
Table 1. Internal calibration results using the 7-peptide calibration mixture

\begin{tabular}{|c|c|c|c|c|c|c|c|c|}
\hline Calibrant & Theor. $m / z$ & Measured $\mathrm{m} / \mathrm{z}$ & $\mathrm{dm}, \mathrm{mDa}$ & $\mathrm{dm}_{\mathrm{r}}, \mathrm{ppm}$ & $\mathrm{dm}(2), \mathrm{mDa}$ & $A_{i}$ & SNR & $R, \times 1000$ \\
\hline Renin inhibitor $2+$ & 513.28199475 & 513.28199425 & -0.00050 & -0.00097 & -0.00343 & 0.639 & 165.3 & 344.4 \\
\hline Bradykinin 2+ & 530.78797558 & 530.78798253 & 0.00695 & 0.01309 & 0.01557 & 1.000 & 258.5 & 346.6 \\
\hline Neurotensin $3+$ & 558.64495321 & 558.64494542 & -0.00779 & -0.01395 & 0.09173 & 0.403 & 139.2 & 300.8 \\
\hline Angiotensin 2+ & 648.84602178 & 648.84602304 & 0.00126 & 0.00193 & 0.00262 & 0.648 & 318.6 & 296.0 \\
\hline Substance P 2+ & 674.37134946 & 674.37134945 & -0.00001 & -0.00002 & 0.07386 & 0.941 & 462.9 & 284.1 \\
\hline Substance P-O 2+ & 682.36880677 & 682.36880069 & -0.00608 & -0.00891 & -0.07713 & 0.295 & 145.3 & 277.0 \\
\hline Fibrinopeptide A 2+ & 768.84988365 & 768.84988116 & -0.00249 & -0.00324 & 0.18179 & 0.901 & 479.3 & 253.1 \\
\hline Neurotensin $2+$ & 837.46379159 & 837.46379617 & 0.00458 & 0.00547 & -0.36193 & 0.278 & 147.9 & 231.5 \\
\hline G-endorphin 2+ & 930.46807927 & 930.46805741 & -0.02186 & -0.02349 & -0.03498 & 0.067 & 35.6 & 206.6 \\
\hline G-endorphin-O 2+ & 938.46553658 & 938.46557525 & 0.03867 & 0.04121 & -0.03616 & 0.214 & 114.0 & 206.1 \\
\hline Renin inhibitor $1+$ & 1025.55671305 & 1025.55669977 & -0.01328 & -0.01294 & 0.16887 & 0.027 & 17.4 & 182.9 \\
\hline
\end{tabular}

Calibrant ions (including oxidized forms $(-0)$ and multiple charge states) obtained from the 7-peptide mixture used for cell characterization. Sample internal calibration results obtained for a single transient, $512 \mathrm{k}$ data points, corresponding to $0.68 \mathrm{~s}$ ion transient duration, $3 \mathrm{~dB}$ excite power attenuation, and low external accumulation time, $T_{\text {acc }}$ of $0.05 \mathrm{~s}$. Internal calibration mass measurement errors, column dm, do not exceed 0.00004 $\mathrm{Da}$, or in relative terms, $\mathrm{dm}_{\mathrm{r}}<0.05 \mathrm{ppm}$. Conditions of increased ion population, $T_{a c c}$ of $0.1 \mathrm{~s}$, produce mass measurement errors up to $0.36 \mathrm{mDa}$ column dm (2). Relative ion abundances $A_{i}$ and signal-to-noise ratios, SNR, correspond to $T_{a c c}$ of $0.05 \mathrm{~s}$. Mass resolving power, $\mathrm{R}$, has been obtained using entire $1 \mathrm{M}$ data points of the same ion transient, acquisition time $1.36 \mathrm{~s}$.

to obtain the signal intensity from each transient segment and then the intensities were fit to the exponential decay profile.

\section{Results and Discussion}

Characterization of the compensated cell used an experimental approach similar to that used to investigate the effect of global and local space charge on mass measurement accuracy across a range of excitation powers and ion populations [32, 45]. Mass spectra were acquired for peptide ions generated by electrospray ionization from the peptide mixture described above. A custom Tk/Tcl script automatically acquired mass spectra with excitation attenuation ranging from 0 to $20 \mathrm{~dB}$ in increments of $0.5 \mathrm{~dB}$. This sequence was repeated consecutively for external accumulation times of 0.05, 0.1 and $0.2 \mathrm{~s}$, effectively covering the useful range of in cell ion populations. This procedure allowed for the two-dimensional (2D) characterization of the instrument in terms of excitation power and ion population in a relatively short time $(30 \mathrm{~min})$. Custom data processing software then produced profiles of total ion current, mass measurement accuracy, transient lifetime, $\mathrm{S} / \mathrm{N}$, resolution, and other characteristics.

The profiles of the TIC, transient life-time $\left(t_{e}\right)$, and the internal calibration mass accuracy are shown in Figure 4 for a range of the ion excitation power attenuation in decibels $(\mathrm{dB})$. Both the open and compensated cells were operated using $4 \mathrm{~V}$ trapping voltage during excitation and detection, and the Bruker excitation parameter of $160 \mu \mathrm{s}$ (lower dB values correspond to lower attenuation and result in higher excitation power). Mass spectra were acquired for external accumulation times $\left(T_{a c c}\right)$ of $0.05,0.1$, and $0.2 \mathrm{~s}$ to observe the effects of changing the ion population. The TIC profiles show a maximum at $7 \mathrm{~dB}$ for the open cell (Figure $4 \mathrm{a}$ ) and $3 \mathrm{~dB}$ for the compensated cell (Figure $4 \mathrm{~d}$ ). The compensated cell demonstrated significant improvement in TIC over the open cell for excitation power range from 7 to $2 \mathrm{~dB}$. Both cell configurations used the same capacitively coupled excitation circuitry and were equivalent in terms of the ion excitation radius. The differences among the TIC profiles resulted from improved performance of the compensated cell at larger ion excitation radii.

The improved TIC profile at larger excitation radii was due to improved coherence of the ion cyclotron motion as demonstrated by the longer ion transient lifetimes $\left(t_{e}\right)$ in the compensated cell (Figure 4e) relative to the open cell (Figure $4 \mathrm{~b}$ ). At an excitation power of 3 $\mathrm{dB}$, the open cell ion transient life-time $\left(t_{e}\right)$ was $0.3 \mathrm{~s}$, while $t_{e}$ was $\sim 1 \mathrm{~s}$ for the compensated cell.

The mass measurement accuracy of the compensated cell (Figure 4f) was improved relative to the open cell (Figure 4c), with the most significant improvement achieved for low ion populations, $T_{a c c}=0.05 \mathrm{~s}$. Minimum mass errors of $0.014 \mathrm{ppm}$ were obtained for the compensated cell at an excitation power of $3 \mathrm{~dB}$ versus $0.18 \mathrm{ppm}$ mass errors obtained for the open cell at $8.5 \mathrm{~dB}$. The mass error obtained for both cell configurations increased with longer external accumulation times $\left(T_{u c c}\right)$ that corresponded to increased ion populations; however the compensated cell showed reduced space-charge related mass errors for excitation power attenuation below the open cell optimum of $7 \mathrm{~dB}$.

A close comparison of selected TIC and mass accuracy profiles for the open and compensated cells reveals systematic differences in the range of increased ion excitation powers, $\mathrm{dB}<6$ (Figure $4 \mathrm{~g}$ and $\mathrm{h}$ ). Ion transients acquired for the two cell configurations for such conditions provide an insight on how the ion cyclotron motion is influenced by the different electric potential distributions.

At $3 \mathrm{~dB}$ attenuation, the ion transients for the open cell (Figure 5a) had a pattern (i.e., beats) typical for a mixture of components with slightly different frequencies, and the corresponding mass spectrum revealed peak splitting, as demonstrated by a sample mass peak 

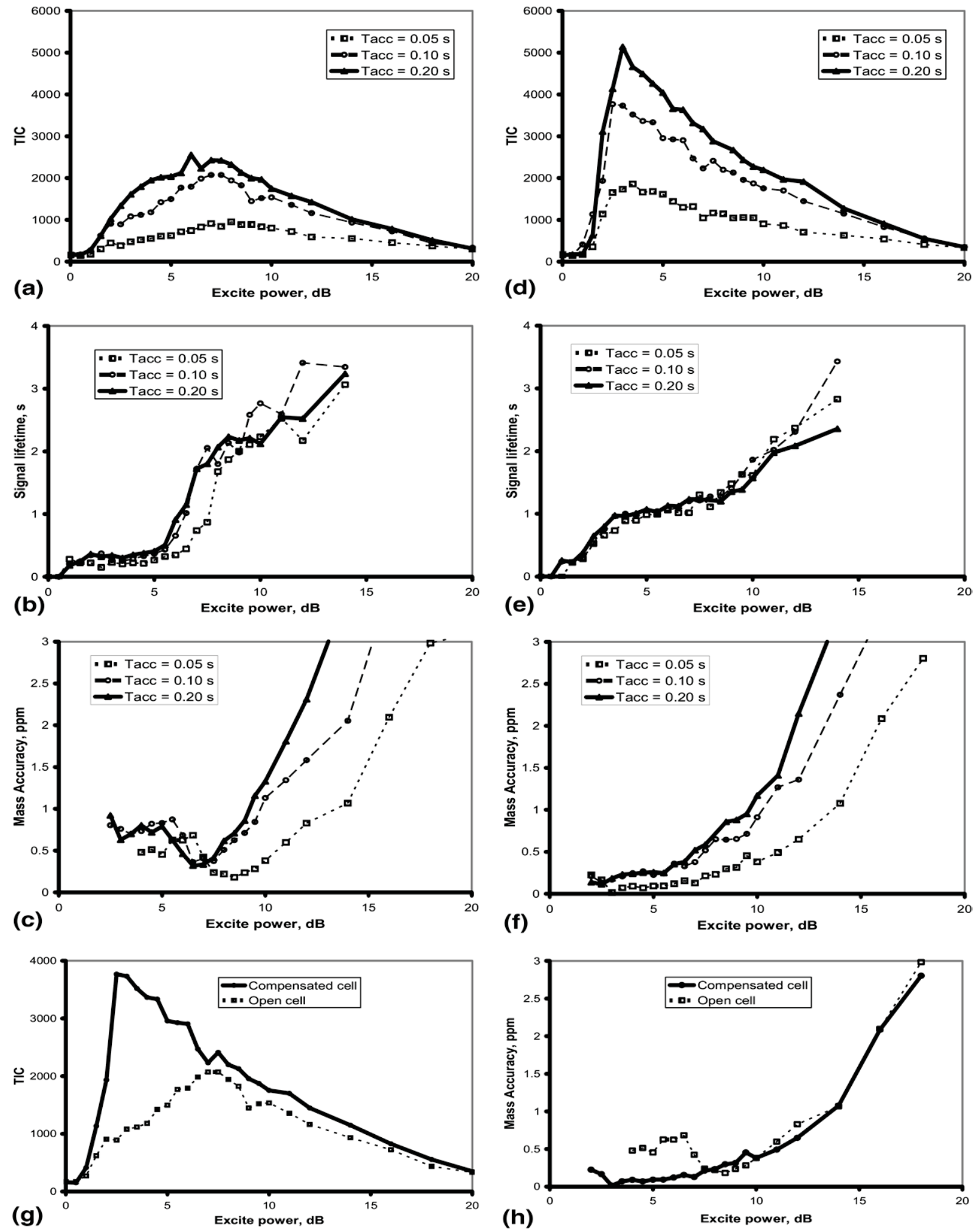

Figure 4. Experimental profiles versus excitation power attenuation, $\mathrm{dB}$, obtained for the two cell potential configurations. Experimental parameters were: direct ESI infusion of the peptide calibration mixture with three external ion accumulation times $\left(T_{a c c}\right)$ of $0.05,0.1$ and $0.2 \mathrm{~s}$; cell trapping voltages during excitation and detection of $4 \mathrm{~V}$; and a Bruker excitation time of $160 \mu \mathrm{s}$. Open cell results: (a) total ion current (TIC), (b) characteristic lifetime of the ion transient, $t_{e}$, and (c) mass accuracy of the internal calibration. Compensated cell results: (d) total ion current (TIC), (e) characteristic lifetime of the ion transient $t_{e}$, and (f) mass accuracy of the internal calibration. Comparison of excitation profiles for the two cell configurations: (g) total ion current (TIC) with $T_{a c c}$ of $0.1 \mathrm{~s}$, and (h) mass measurement accuracy of the internal calibration from (c) and (f), for $T_{a c c}$ of $0.05 \mathrm{~s}$. 


\section{Open cell, no compensation}
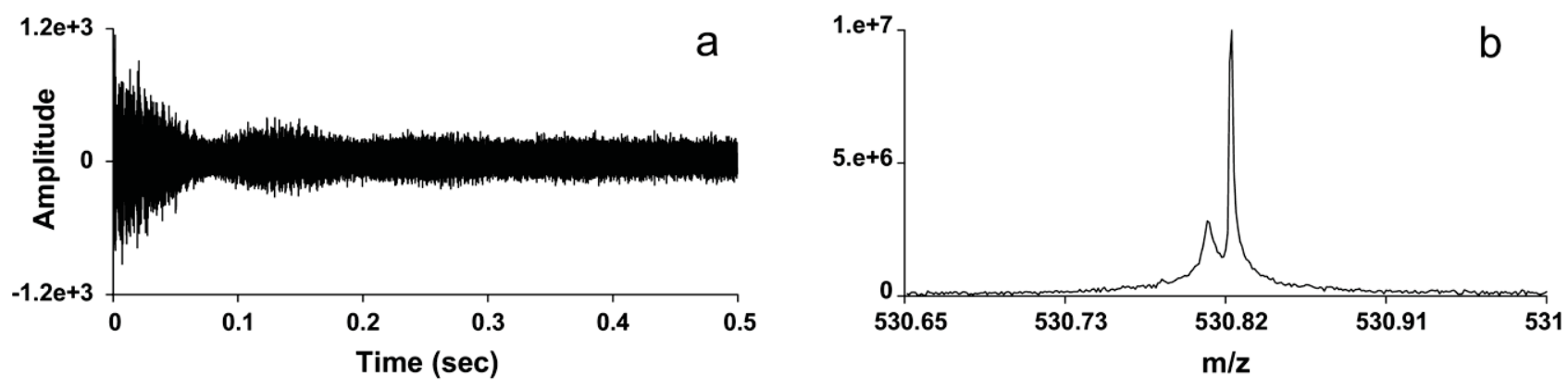

Compensated cell
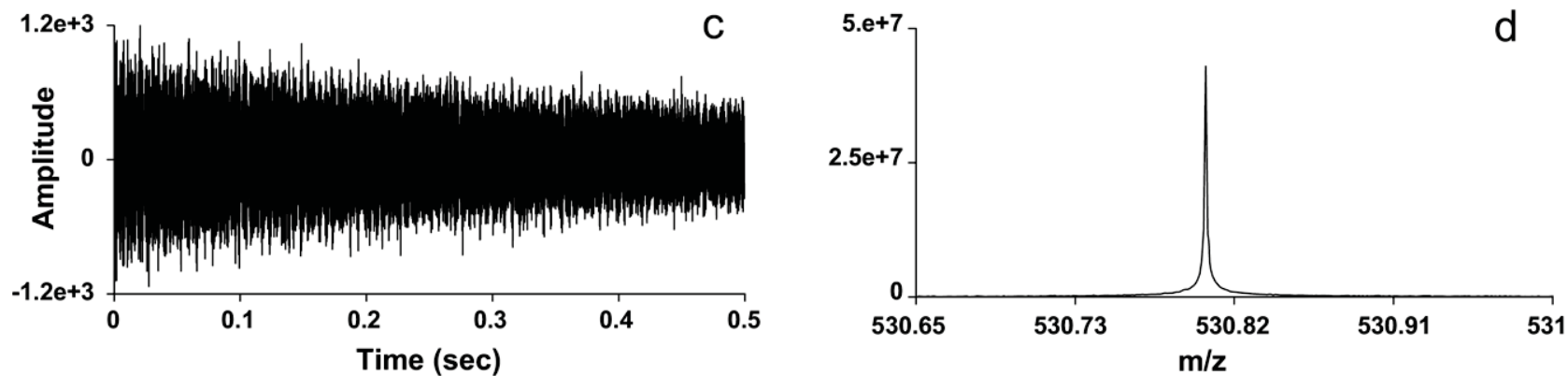

Figure 5. Ion transients and peak shapes obtained using an excitation power of $3 \mathrm{~dB}$, which corresponds to TIC and mass accuracy optimum for the compensated cell: (a) sample ion transient for the open cell configuration, (b) sample peak from Fourier-transformed data for the open cell configuration, (c) sample ion transient for the compensated cell configuration, (d) sample peak from Fourier-transformed data for the compensated cell configuration.

in Figure $5 \mathrm{~b}$. The transient obtained with the compensated cell (Figure 5c) had a uniform decay profile, and the corresponding transformed peak (Figure $5 \mathrm{~d}$ ) had no apparent splitting. This result reproduces an observation reported for the first experimentally tested harmonized ICR cell, where the peak splitting observed with the cubic cell at the excitation radius $\sim 0.75$ of the maximum radius was not visible with the matrix shimmed cell with mass resolution $\sim 8000$ at $\mathrm{m} / \mathrm{z} 186$, tentatively attributed to Coulomb repulsions resulting from too many ions in the trap [22]. The mass resolving power obtained in our measurements approached theoretical limit for low-pressure line shape, $\sim 400,000$ at $\mathrm{m} / \mathrm{z} 530$ (see below).

The compensation design used in this work does not provide linearization of the dipolar excitation and detection and, thus, additional harmonic peaks were not suppressed, as it was demonstrated with the matrix shimmed cell [22]. Experiments confirmed slightly increased harmonic peaks when working at increased excitation radii, e.g., at $3 \mathrm{~dB}$ attenuation the intensity of harmonic peaks was $\sim 50 \%$ higher compared with $6 \mathrm{~dB}$ conditions. The presence of harmonics did not create a problem since the peaks were automatically rejected by the deisotoping processing. Generally, the location of harmonic peaks can be accurately predicted and the peaks can be removed in a post-processing step, with minor or no interference to identification of useful peaks, thanks to the high spectral resolution.

A comparison of TIC (Figure 4g) and mass accuracy (Figure $4 \mathrm{~h}$ ) versus excitation power for the open and compensated cell configurations produced similar results for excitation attenuation above $7 \mathrm{~dB}$, which indicates good stability and reproducibility of the measurements. At higher excitation powers of 7 to $3 \mathrm{~dB}$, the compensated cell produced gradually increasing signal intensity and improved mass measurement accuracy, while TIC and mass accuracy decreased for the open cell. Similar trends were observed for trapping voltages from 0.5 to $16 \mathrm{~V}$ and for the Bruker excitation time parameter from 80 to $320 \mu \mathrm{s}$.

The different responses of the open and compensated cells to excitation power can be interpreted in terms of magnetron frequency shifts (as considered in the Cell Design section). The excitation $\mathrm{dB}$ values are related to the postexcitation cyclotron radius via the following relationship: 


$$
R_{\text {excite }}(d B)=R_{\max } 10^{\left(d B_{0}-d B\right) / 20}
$$

Here $R_{\max }$ represents the maximum excitation radius that corresponds to the loss of ions on the cell electrode; the excitation power corresponding to $R_{\max }$, was $d B_{0} \approx$ $0.5 \mathrm{~dB}$, according to TIC profiles in Figure $4 \mathrm{~g}$. As an approximation, one can assume $R_{\max }$ equal to the cell radius $r_{0}$, and neglect contributions from the magnetron radius and ion cloud radial size. The use of eq 3 and 3 $\mathrm{dB}$ (i.e., the optimal excitation power for the compensated cell) resulted in a calculated ion excitation radius of $0.75 r_{0}$.

The radial potential plot at a distance of 0.75 from the cell axis showed pronounced nonuniformity of the radial field for the open cell configuration (Figure $3 b$ ), which leads to variations of the effective cyclotron frequency of about $20 \mathrm{ppm}$, as estimated above. This estimation is roughly consistent with the peak splitting observed in the open cell at $3 \mathrm{~dB}$ excitation power (Figure 5b). The compensated cell had $10 \times$ lower deviations from the ideal potential, which resulted in a more uniform radial field profile (Figure $3 \mathrm{~d}$ ), and the corresponding frequency variations were not visible in the experimental peak shape (Figure $5 \mathrm{~d}$ ). The smaller axial variations of the magnetron frequency resulted in $\mathrm{CO}^{-}$ herent ion cyclotron motion for a longer time scale of $\sim 1 \mathrm{~s}$, which explains the difference in ion transients for the open and compensated cell configurations (Figure $5 \mathrm{a}$ and $\mathrm{c}$ ).

Reduced excitation power (i.e., attenuation $>7 \mathrm{~dB}$ ) corresponded to excitation radii less than $0.5 r_{0}$, where the open cell anharmonicity was reduced, as seen from the $E_{r} / r$ profile for $0.5 r_{0}$ (Figure $3 \mathrm{~b}$ ), and thus the similar TIC profiles for open and compensated cells (Figure $4 \mathrm{~g}$ ). When only a short initial interval of the ion transient $\Delta t$ $\sim 0.1 \mathrm{~s}$ was used for the TIC calculation, the open cell TIC continued to increase up to $3 \mathrm{~dB}$, similar to the compensated cell behavior, which confirmed that shorter transients rather than smaller initial signal intensity was the factor behind TIC decline for the open cell at excitation radii above $0.5 r_{0}$.

The mass measurement errors increased for reduced excitation powers (i.e., attenuation above $7 \mathrm{~dB}$ ) for both the open and compensated cells, which is consistent with increased space charge effects due to a more compact configuration of rotating ion clouds. A reduction in attenuation to below $6 \mathrm{~dB}$ leads to a gradual improvement of the mass accuracy for the compensated cell below $0.1 \mathrm{ppm}$ in the range of 3 to $5 \mathrm{~dB}$ for small ion population with $T_{a c c}$ of $0.05 \mathrm{~s}$ (Figure $4 \mathrm{~h}$ ). The open cell configuration exhibited the opposite trend; mass errors increased to $0.6 \pm 0.1 \mathrm{ppm}$ in the range 4 to $7 \mathrm{~dB}$, which is consistent with increased magnetron frequency shifts that caused deviations from the calibration law.

The relatively low ion population corresponding to $T_{u c c}$ of $0.05 \mathrm{~s}$ lead to minimal space charge related frequency perturbations, which explained the very good mass accuracy achievable for optimal excitation conditions (Figure 4h). The compensated cell main- tained coherent ion motion at increased postexcitation radii, which further reduced space charge effects. The mass accuracy improvement was reproducibly observed for excitation powers that corresponded to excitation radii near 0.7 of the cell radius. Table 1 lists the internal calibration details from mass spectra with an excitation power of $3 \mathrm{~dB}$ and external accumulation times of 0.05 and $0.1 \mathrm{~s}$, using 11 calibrants that cover a $\mathrm{m} / \mathrm{z}$ range from 513 to 1026 and a dynamic range from 17 to 479 (units of signal-to-noise ratio, SNR column). The mass measurement errors with an external accumulation time of $0.05 \mathrm{~s}$ were below $0.00004 \mathrm{Da}$, or in terms of relative deviation $\left(d m_{r}\right)$ below $0.05 \mathrm{ppm}$, and the RMS mass error for all 11 calibrants was $0.016 \mathrm{ppm}$. Remarkably, the accurate mass measurements were obtained in the high-throughput mode, using single transients, $0.68 \mathrm{~s}$ acquisition time, and no elaborate procedures such as multistage ion cooling or trapping potential manipulations.

The mass resolving power (last column of Table 1) closely approaches the theoretical maximum for FT-ICR line shape in the low-pressure limit [46], with a resolution of 390,000 at $m / z 530.8$ and an acquisition time of $1.36 \mathrm{~s}$. The resolution did not depend noticeably on the external accumulation time, indicating negligible Coulomb repulsions, or space charge effects. This observation confirms that the compensated cell maintained near ideal coherent ion motion at excitation radii $\sim 0.7 r_{0}$.

Increased ion populations with $T_{a c c}$ of 0.1 and $0.2 \mathrm{~s}$ lead to increased mass measurement errors (Figure $4 \mathrm{c}$ and $\mathrm{f}$ ), which is consistent with the general trend observed in FT-ICR MS. A sample single transient from the compensated cell with $T_{a c c}$ of $0.1 \mathrm{~s}$ resulted in a $\times 2$ increase in ion signal and correspondingly, an increased base peak dynamic range $\sim 1000$ in SNR units. Individual errors [column dm (2) in Table 1] were within 0.0004 $\mathrm{Da}$, and RMS of all 11 mass measurement errors increased to $0.17 \mathrm{ppm}$. Importantly, the conditions of increased ion populations resulted in a twofold improvement in mass accuracy in the compensated cell compared with the open cell, due to the larger achievable excitation radii and correspondingly lower space charge related perturbations of the calibration law.

To further characterize the utility of the new cell for high-throughput proteomics measurements, we have conducted LC-MS measurements with a standard mixture of proteins digested with trypsin. The mass accuracy and coverage of each analysis can be characterized by a mass accuracy histogram, Figure 6 [29]. The infinity cell data were acquired with the same front end configuration, just before replacing the infinity cell with the new cell. It should be noted that the current front end configuration is not optimized for the tryptic peptide LC-MS measurements and uses no gain control, so the mass accuracy obtained from such measurements is determined by the ion population. For comparison, we are using conditions for each of the configurations, including the external accumulation time $(0.1 \mathrm{~s})$ and sample concentration $(0.044 \mathrm{mg} / \mathrm{mL})$, which produced 


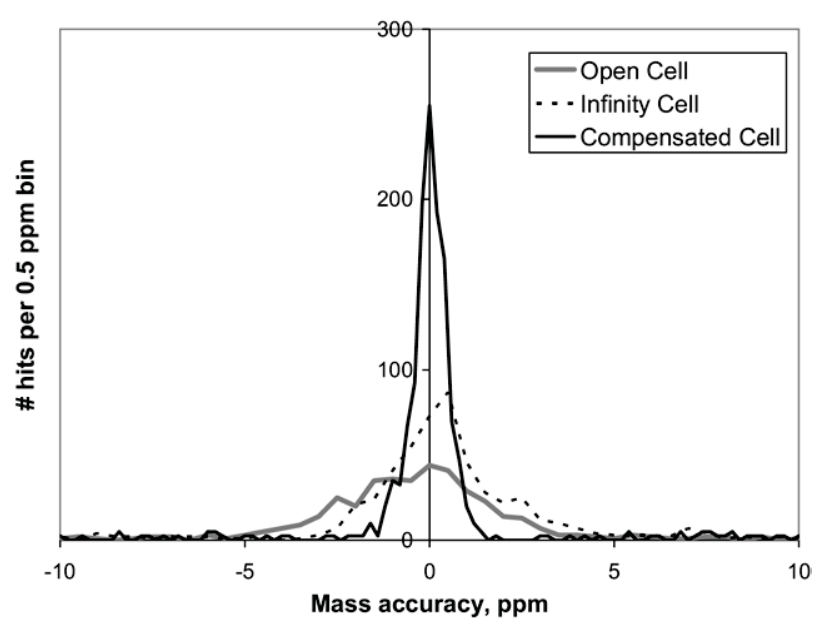

Figure 6. Mass accuracy histograms obtained for LC-MS separation of the standard mixture of proteins digested with trypsin, number of matched elution features per mass residual bin of 0.5 ppm, see text. Open cell, compensation is not applied, gray curve: 3.6 ppm FWHM, 7.6 ppm baseline, 356 hits. Infinity cell, dotted curve: 2.2 ppm FWHM, 5.8 ppm baseline, 446 hits. Compensated cell, black curve: 0.9 ppm FWHM, 2.1 ppm baseline, 465 hits (bin reduced to $0.2 \mathrm{ppm}$, with $\mathrm{y}$-scale adjusted $\times 2.5$ to compensate for the different bin size).

the optimal number of identifications, $\sim 400$ peptide mass tags. The compensated cell produced similar or better mass tag coverage, with better mass measurement precision, below 1 ppm at FWHM of the histogram peak. The baseline mass accuracy improved from 6-8 ppm for the infinity cell and the open cell to $2 \mathrm{ppm}$ for the compensated cell. The baseline mass accuracy determines the optimal mass accuracy tolerance for bioapplications and is critical for improving the identification confidence [29]. This initial test showed a better FWHM mass precision, $0.9 \mathrm{ppm}$, than reported in the recent study on mass measurement accuracy of our proteomics data production FTICR instruments, e.g., ح ppm FWHM for 11 tesla FT-ICR before recalibration [29]. LC-MS data for $10 \times$ diluted solution produced 164 hits with 0.7 ppm FWHM and 1.3 ppm baseline accuracy, indicating that space charge effects produced a major contribution to observed mass errors and optimization of the front end should produce further mass accuracy improvement.

\section{Conclusion}

We developed a new FT-ICR trapped ion cell with a DC trapping potential closely approaching that of an ideal Penning trap, i.e., 3D axial quadrupolar potential distribution. The new cell builds on the now common open cylindrical geometry, supplemented with two pairs of cylindrical compensation segments, making the total number of segments equal to 7 . Each segment was split into four sectors, and all 28 sectors were involved in the capacitively coupled excite/detect circuit, similar to the regular capacitively coupled open cylindrical cell. $\mathrm{Nu}-$ merical calculations, aimed at minimization of spatial variations of the radial electric field divided by radius, produced dimensions and compensation voltages that deliver an order-of-magnitude suppression of these variations, ensuring practically constant effective ion cyclotron frequency for ions occupying various radial and axial positions. The new cell can be easily installed in place of a standard ICR cell and is less involved, both mechanically and electrically, than harmonized cells reported previously, the matrix shimmed cubic cell having 150 elements [22], and the 32 pole cell having 32 poles plus 15 cylindrical segments [24].

Experimental study of the new cell aimed at its utility for proteomics applications that require high mass measurement accuracy and coverage in a robust high throughput LC-FT-ICR platform. The capability of the shimmed cell to produce mass spectra without peak splitting at increased ion cyclotron radii, 0.7 of the cell radius, first reported for the matrix shimmed cell [22], was reproduced in the present study, now with the mass resolving power not compromised by the space charge effects.

Performance optimization covering a range of excitation powers and external accumulation times confirmed advantages of the new cell both in improved mass measurement accuracy and in higher achievable ion signals. Mass accuracy tests using 11 calibrant ions covering a wide $\mathrm{m} / \mathrm{z}$ range, 513 to 1025 , showed 0.01 ppm level deviations from the calibration law, reproducibly obtained for low ion populations and optimally high excitation radii. The accurate mass measurements were obtained in the high-throughput mode, using single $0.68 \mathrm{~s}$ transients without additional time-consuming procedures, such as multistage ion cooling or trapping potential manipulations.

\section{Acknowledgments}

The authors gratefully acknowledge D. C. Prior, J. R. Ewing, G. A. Anderson, and H. R. Udseth for help with the cell design, and C. D. Masselon and A. N. Vilkov for discussions of the FT-ICR cell harmonicity issues, M. E. Belov for reading the manuscript and providing helpful suggestions. Portions of this work were supported by the National Center for Research Resources (RR 018522), the National Institute of Allergy and Infectious Diseases (NIH/ DHHS through interagency agreement Y1-AI-4894-01), the National Institute of General Medical Sciences (NIGMS, R01 GM063883), and the U.S. Department of Energy (DOE) Office of Biological and Environmental Research. Work was performed in the Environmental Molecular Science Laboratory, a DOE national scientific user facility located on the campus of Pacific Northwest National Laboratory (PNNL) in Richland, Washington. PNNL is a multi-program national laboratory operated by Battelle for the DOE under contract DE-AC05-76RLO 1830.

\section{References}

1. Comisarow, M. B.; Marshall, A. G. Fourier-Transform Ion-Cyclotron Resonance Spectroscopy. Chem. Phys. Lett. 1974, 25, 282-283.

2. Marshall, A. G. Milestones in Fourier Transform Ion Cyclotron Resonance Mass Spectrometry Technique Development. Int. J. Mass Spectrom. 2000, 200, 331-356. 
3. Marshall, A. G.; Hendrickson, C. L. Fourier Transform Ion Cyclotron Resonance Detection: Principles and Experimental Configurations. Int. J. Mass Spectrom. 2002, 215, 59-75.

4. Smith, R. D.; Anderson, G. A.; Lipton, M. S.; Pasa-Tolic, L.; Shen, Y.; Conrads, T. P.; Veenstra, T. D.; Udseth, H. R. An Accurate Mass Tag Strategy for Quantitative and High-Throughput Proteome Measurements. Proteomics 2002, 2, 513-523.

5. Aebersold, R.; Mann, M. Mass Spectrometry-Based Proteomics. Nature 2003, 422, 198-207.

6. Page, J. S.; Masselon, C. D.; Smith, R. D. FTICR Mass Spectrometry for Qualitative and Quantitative Bioanalyses. Curr. Opin. Biotechnol. 2004, 15, 3-11.

7. Paša-Tolić, L.; Masselon, C.; Barry, R. C.; Shen, Y; Smith, R. D. Proteomic Analyses Using an Accurate Mass and Time Tag Strategy. Biotechniques 2004, 37(4), 621-639.

8. Bogdanov, B.; Smith, R. D. Proteomics by FTICR Mass Spectrometry: Top Down and Bottom Up. Mass Spectrom. Rev. 2005, 24, 168

9. Hawkridge, A. M.; Nepomuceno, A. I.; Lovik, S. L.; Mason C. J.; Muddiman, D. C. Effect of Postexcitation Radius on Ion Abundance, Mass Measurement Accuracy, and Isotopic Distributions in Fourier Transform Ion Cyclotron Resonance Mass Spectrometry. Rapid Commun. Mass Spectrom. 2005, 19, 915-918.

10. Frahm, J. L.; Capo Velez, C. M.; Muddiman, D. C. Understanding the Influence of Postexcite Radius and Axial Confinement on Quantitative Proteomic Measurements Using Fourier Transform Ion Cyclotron Resonance Mass Spectrometry. Rapid Commun. Mass Spectrom. 2007, 21, 1196-1204.

11. Caravatti, P.; Allemann, M. The Infinity cell-a New Trapped-Ion Cell with Radiofrequency Covered Trapping Electrodes for FourierTransform Ion-Cyclotron Resonance Mass-Spectrometry. Org. Mass Spectrom. 1991, 26(5), 514-518.

12. Beu, S. C.; Laude, D. A. Elimination of Axial Ejection During Excitation with a Capacitively Coupled Open Trapped-Ion Cell For FourierTransform Ion-Cyclotron Resonance Mass-Spectrometry. Anal. Chem. 1992, 64(2), 177-180.

13. Vartanian, V. H.; Laude, D. A. Optimization of a Fixed-Volume Open Geometry Trapped Ion Cell for Fourier-Transform Ion-Cyclotron MassSpectrometry. Int. J. Mass Spectrom. 1995, 141(3), 189-200.

14. Guan, S. H.; Marshall, A. G. Ion Traps For Fourier-Transform IonCyclotron Resonance Mass-Spectrometry-Principles and Design of Geometric and Electric Configurations. Int. J. Mass Spectrom. 1995, 146, 261-296.

15. Gabrielse, G. Relaxation Calculation of the Electrostatic Properties of Compensated Penning Traps with Hyperbolic Electrodes. Phys. Rev. A 1983, 27, 2277-2290.

16. Gabrielse, G.; MacKintosh, F. C. Cylindrical Penning Traps with Orthogonalized Anharmonicity Compensation. Int. J. Mass Spectrom. Ion Processes 1984, 57, 1-17.

17. Gabrielse, G.; Haarsma, L.; Rolston, S. L. Open-Endcap Penning Traps for High-Precision Experiments. Int. J. Mass Spectrom. 1989, 88(2/3), 319-332.

18. Naito, Y.; Fujiwara, M.; Inoue, M. Improvement of the Electric Field in the Cylindrical Trapped-Ion Cell. Int. J. Mass Spectrom. Ion Processes 1992, 120, 179-192.

19. Vartanian, V. H.; Laude, D. A. Optimization of a Fixed-Volume Open Geometry Trapped Ion Cell for Fourier Transform Ion Cyclotron Mass Spectrometry. Int. I. Mass Spectrom. Ion Processes 1995, 141, 189-200.

20. Vartanian, V. H.; Hadjarab, F.; Laude, D. A. Open Cell Analog of the Screened Trapped-Ion Cell Using Compensation Electrodes for Fourier Transform Ion Cyclotron Resonance Mass Spectrometry. Int. J. Mass Spectrom. 1995, 151(2/3), 175-187.

21. Knobeler, M.; Wanczek, K. P. Theoretical Investigation of Improved Ion Trapping in Matrix-Assisted Laser Desorption/Ionization Fourier Transform Ion Cyclotron Resonance Mass Spectrometry: Independence of Ion Initial Velocity. Int. J. Mass Spectrom. Ion Processes 1997, 163, $47-68$

22. Jackson, G. S.; White, F. M.; Guan, S. H.; Marshall, A. G. MatrixShimmed Ion Cyclotron Resonance Ion Trap Simultaneously Optimized for Excitation, Detection, Quadrupolar Axialization, and Trapping. J. Am. Soc. Mass Spectrom. 1999, 10(8), 759-769.

23. Fei, X.; Snow, W. M. Cylindrical Penning Traps with Dynamic Orthogonalized Anharmonicity Compensation for Precision Experiments. Nucl. Instrum. Methods Phys. Res. A 1999, 425, 431-440.

24. Bruce, J. E.; Anderson, G. A.; Lin, C. Y.; Gorshkov, M.; Rockwood, A. L.; Smith, R. D. A Novel High-Performance Fourier Transform Ion Cyclotron Resonance Cell for Improved Biopolymer Characterization. J. Mass Spectrom. 2000, 35, 85-94.
25. Barlow, S. E.; Tinkle, M. D. Linearizing an Ion Cyclotron Resonance Cell. Rev. Sci. Instrum. 2002, 73(12), 4185-4200.

26. Gooden, J. K.; Rempel, D. L.; Gross, M. L. Evaluation of Different Combinations of Gated Trapping, RF-Only Mode, and Trap Compensation for In-Field MALDI Fourier Transform Mass Spectrometry. JASMS 2004, 15, 1109-1115.

27. Rempel, D. L.; Gross, M. L. Compensation Designs for Cylindrical Traps in FTMS that Permit Improved Coulombic Dynamic Range. Proceedings of the 53rd ASMS Conference; San Antonio, TX, June, 2005; on CD ROM.

28. Tolmachev, A. V.; Smith, R. D. A Multiregion Recalibration Approach for Obtaining ppm-Range Mass Measurement Accuracy for LC-MS Analyses of Complex Proteomics Mixtures. Proceedings of the 54th ASMS Conference; Seattle, WA, May, 2006; on CD ROM.

29. Tolmachev, A. V.; Monroe, M. E.; Jaitly, N.; Petyuk, V. A.; Adkins, J. N.; Smith, R. D. Mass Measurement Accuracy in Analyses of Highly Complex Mixtures Based Upon Multidimensional Recalibration. Anal. Chem. 2006, 78(24), 8374-8385.

30. Tolmachev, A. V.; Udseth, H. R.; Smith, R. D. The Charge Capacity Limitations of Radio Frequency Ion Guides in Their Use for Improved Ion Accumulation and Trapping in Mass Spectrometry. Anal. Chem. 2000, 72, 970-978.

31. Tolmachev, A. V.; Udseth, H.; Smith, R. D. Modeling the Ion Density Distribution in Collisional Cooling RF Multipole Ion Guides. Int. J. Mass Spectrom. 2003, 222, 155-174.

32. Masselon, C.; Tolmachev, A. V.; Anderson, G. A.; Harkewicz, R.; Smith, R. D. Mass Measurement Errors Caused by "Local" Frequency Perturbations in FTICR Mass Spectrometry. J. Am. Soc. Mass Spectrom. 2002, 13(1), 99-106.

33. Vartanian, V. H.; Laude, D. A. Motional Averaging of Ions for Control of Magnetron Motion in Fourier Transform Ion Cyclotron Resonance Open-Geometry Trapped-Ion Cells. Int. J. Mass Spectrom. 1998, 178(3), 173-186.

34. Easterling, M. L.; Mize, T. H.; Amster, I. J. Routine Part-per-Million Mass Accuracy for High-Mass Ions: Space-Charge Effects in MALDI FT-ICR. Anal. Chem. 1999, 71, 624-632.

35. Taylor, P. K.; Amster, I. J. Space Charge Effects on Mass Accuracy for Multiply Charged Ions in ESI-FTICR. Int. J. Mass Spectrom. 2003, 222, 351-361.

36. Wieghaus, A.; Froehlich, U.; Malek, R.; Horning, S. The Grid Cell: A New Cell Design for Reduced z-Axis Ejection in FTICR MS. Proceedings of the 54th ASMS Conference; Seattle, WA, May, 2006; on CD ROM.

37. Ledford, E. B., Jr.; Rempel, D. L.; Gross, M. L. Space Charge Effects in Fourier Transform Mass Spectrometry Mass Calibration. Anal. Chem. 1984, 56, 2744-2748.

38. Shi, S. D. H.; Drader, J. J.; Freitas, M. A.; Hendrickson, C. L.; Marshall, A. G. Comparison and Interconversion of the Two Most Common Frequency-to-Mass Calibration Functions for Fourier Transform Ion Cyclotron Resonance Mass Spectrometry. Int. J. Mass Spectrom. 2000, 195, 591-598.

39. Francl, T. J.; Sherman, M. G.; Hunter, R. L.; Locke, M. J.; Bowers, W. D.; McIver, R. T. Experimental Determination of the Effects of Space Charge on Ion Cyclotron Resonance Frequencies. Int. J. Mass Spectrom. Ion Processes 1983, 54, 189-199.

40. Van Dyck, R. S., Jr.; Wineland, D. J.; Ekstrom, P. A.; Dehmelt, H. G. High Mass Resolution with a New Variable Anharmonicity Penning Trap. Appl. Phys. Lett. 1976, 28, 446-448.

41. Van Dyck, R. S., Jr.; Schwinberg, P. B. Preliminary Proton/Electron Mass Ratio Using a Compensated Quadring Penning Trap. Phys. Rev. Lett. 1981, 47, 395-398.

42. Easterling, M. L.; Pitsenberger, C. C.; Amster I. J. RF Capacitive Coupling with Efficient Gated Trapping in Internal Matrix-Assisted Laser Desorption Ionization Fourier Transform Ion Cyclotron Resonance. J. Am. Soc. Mass Spectrom. 1997, 8(2), 195-198.

43. Guoa, X.; Duursmaa, M.; Al-Khalili, A.; McDonnell, L. A.; Heeren, R. M. A. Design and Performance of a New FT-ICR Cell Operating at a Temperature Range 77-438 K. Int. J. Mass Spectrom. 2004, 231, 37-45.

44. Sharma, S.; Simpson, D. C.; Tolić, N.; Jaitly, N.; Mayampurath, A. M. Smith, R. D.; Paša-Tolić, L. Proteomic Profiling of Intact Proteins Using WAX-RPLC 2-D Separations and FTICR Mass Spectrometry. J. Proteome Res. 2007, 6(2), 602-610.

45. Tolmachev, A. V.; Masselon, C. D.; Anderson, G. A.; Paša-Tolić, L. Udseth, H. R.; Smith, R. D. Physical and Mathematical Frequency Shifts in FTICR Spectra. Proceedings of the 50th ASMS Conference on Mass Spectrometry and Allied Topics; Orlando, FL, June, 2002; on CD-ROM.

46. Comisarow, M. B.; Marshall, A. G. Theory of Fourier Transform Ion Cyclotron Resonance Mass Spectrometry. I. Fundamental Equations and Low-Pressure Line Shape. J. Chem. Phys. 1976, 64, 110-119. 